\title{
An assessment of the interoperability of PPP-AR network products
}

\author{
Garrett Seepersad ${ }^{*}$ iD and Sunil Bisnath
}

\begin{abstract}
Integer ambiguity resolution of carrier-phase measurements from a single receiver can be implemented by applying additional satellite corrections (products) to mitigate unmodelled satellite equipment delays. Interoperability of different PPP-AR products would allow the PPP user to transform independently generated PPP-AR products to obtain multiple fixed solutions of comparable precision and accuracy with limited changes required to user PPP measurement processing software. The ability to provide multiple solutions would increase the reliability of the solution for, e.g., real-time processing; if there were an outage in the generation of one set of PPP-AR products, the user could instantly switch streams to a different provider.

There are currently three main public providers of real-time products that enable PPP-AR. These include School of Geodesy and Geomatics at Wuhan University (SGG-WHU), Natural Resources Canada (NRCan) and Centre National d'Etudes Spatiales (CNES). The presented research examines the PPP-AR products generated from the FCB (Fractional Cycle Bias) model and IRC (Integer Recovery Clock) model that have been transformed into the DC (Decoupled Clock) format and applied within the PPP user solution. Interoperability of the different PPP-AR products is a challenging task due to the public availability of different quality of products, limited literature documenting the conventions adopted within the network solution of the providers and unclear definitions of the corrections. The novelty of the research is in the analysis of using the transformed products. The convergence time (time to first fix and time to a pre-defined performance level), position precision (repeatability), position accuracy and solution outliers are examined. Equivalent performance was noted utilizing the different methods. Of the four solutions, FCB products had the highest accuracy. This is attributed to the products being generated using final IGS orbit and clock products. To confirm this, FCBs generated using GRG orbit and clock products were also examined and comparable performance was observed between the FCBs and IRC (GRG) products. The least accurate solution was obtained using the IRC (CNT) products, which was due to the products being archived real time products.
\end{abstract}

\section{Introduction}

Precise Point Positioning (PPP) requires a relatively long initialization period of at least a few tens of minutes for the carrier-phase ambiguities to converge to constant values and for the solution to reach its optimal precision. The carrier-phase signals are approximately two orders of magnitude more precise than the primary pseudorange (code) signals. However, measurements of the carrierphases are ambiguous, relative to those of the pseudoranges by an unknown number of integer cycles. In RTK, the integer nature of the carrier-phase ambiguities is uncovered by explicitly differencing simultaneous observations from multiple stations visible to the same satellites. Differencing

* Correspondence: GSeeper@yorku.ca

York University, Toronto, Canada of simultaneous observations can be thought of as an optimal correction method (Collins and Bisnath 2011) as the error sources are not modelled. Ambiguity resolution in PPP (PPP-AR) requires the equipment delays within the GPS measurements to be mitigated, which would allow for resolution of the integer nature of the carrier-phase measurements (Laurichesse and Mercier 2007; Collins 2008; Mervart et al. 2008; Ge et al. 2008; Teunissen et al. 2010; Bertiger et al. 2010; Geng et al. 2012; Lannes and Prieur 2013). Resolution of these ambiguities convert the carrier-phases into precise pseudorange measurements, with measurement noise at the centimetreto-millimetre level compared to the metre-to-decimetrelevel of the direct pseudoranges (Collins et al. 2010). If the ambiguities could be isolated and estimated as integers, then there would be more information that could be 
exploited to accelerate convergence to give cm-level horizontal accuracy within an hour of data collection. Collins et al. (2008) and Laurichesse et al. (2009) saw improvements in hourly position estimates by $2 \mathrm{~cm}$ and Geng et al. (2010) saw noticeable hourly improvements from $1.5,3.8$ and $2.8 \mathrm{~cm}$ to $0.5,0.5,1.4 \mathrm{~cm}$ for north, east and up, respectively.

Integer ambiguity resolution of measurements from a single receiver can be implemented by applying additional satellite products, where the fractional component, representing the satellite equipment delay, has been separated from the integer ambiguities in a network solution. One method of deriving such products is to estimate the satellite equipment delay by averaging the fractional parts of the steady-state float ambiguity estimates (Ge et al. 2008), and the other is to estimate the pseudorange and carrierphase clocks independently by fixing the undifferenced ambiguities to integers in advance (Collins 2008; Laurichesse et al. 2009). The initial application of ambiguity resolution to PPP was made by Ge et al. (2008) using the Uncalibrated Phase Delay (UPD) model, now called Fractional Cycle Bias (FCB) model (Geng et al. 2010, 2012). The FCB method estimates combined pseudorange/ carrier-phase satellite equipment delays removing the pseudorange satellite equipment delays from common clock estimates, such as those provided by the IGS (International GNSS Service). Common clock implies the estimated satellite clock correction is applied to both pseudorange and carrier phase measurements. An alternative approach to PPP-AR was developed by Collins et al. (2008) called the Decoupled Clock (DC) model. The underlying concept of the DC model is the carrier-phase and pseudorange measurements are not synchronized with each other at the level of precision of the carrier-phase. The timing of the different observable must be considered separately, if they are to be processed together rigorously. The DC model is a reformulation of the ionosphere-free pseudorange and carrier-phase observation equations for GPS, when combined with the wide lane carrier-phase and narrow lane pseudorange observable, permits ambiguity resolution of ambiguities (Collins 2008). The Integer Recovery Clocks (IRC) model presented by Mercier and Laurichesse (2007) consists of daily averages of the wide lane biases and carrier-phase clocks. Zhang et al. (2011) presented a different approach to PPP-AR where the user is provided with the satellite equipment delay for the L1 and L2 carrierphase signals. In Teunissen and Khodabandeh (2015), the model presented by Zhang et al. (2011) is referred to as the Common Clock (CC-1).

Similar positioning performances have been demonstrated amongst the three methods, DC (Collins et al. 2010), FCB (Ge et al. 2008; Geng et al. 2009) and IRC (Laurichesse et al. 2009). Studies such as Geng et al. (2010), Shi and Gao (2013), and Teunissen and Khodabandeh
(2015) have identified the differences and the fundamental similarities between the methods. For the PPP user, the mathematical model is similar; the different PPP-AR products contain the same information and as a result would allow for a one-to-one transformations, allowing interoperability of the PPP-AR products (Teunissen and Khodabandeh 2015). The advantage of interoperability of the different PPP-AR products would be to allow the PPP user to transform independently generated PPP-AR products to obtain multiple fixed solutions of comparable precision and accuracy. The ability to provide multiple solutions would increase the reliability of the solution for, e.g., realtime processing; if there were an outage in the generation of the PPP-AR products, the user can instantly switch streams to a different provider. Interoperability also implies that the user can switch providers within the same PPP engine. The strategy of integrating interoperability of the different products would be dependent on the user application and coding infrastructure of the PPP engine. The research presented examines the PPP-AR products generated from the FCB and IRC model that have been transformed into the DC format and applied within the PPP user solution. The novelty of the research is the solution analysis using the transformed product. The convergence time (time to first fix and time to a pre-defined performance level), position precision (repeatability), position accuracy and solution outliers would be examined. The temporal and spatial behaviour of these estimated terms is examined for the various products applied to understand the unmodelled effects that introduced incorrect solution fixes.

\section{Overview PPP-AR techniques and products}

The standard GPS dual-frequency pseudorange and carrier-phase observation equations are presented in Eqs. ( 1 and 2). Where $i$ denotes the frequency dependent GPS measurements frequencies $L 1$ or $L 2$. ps represents the single difference satellites, where $p$ represents the reference satellite, $s$ represents the other tracked satellite and $u$ represents the user position.

$$
\begin{aligned}
& \Delta \phi_{u, i}^{p s}=\Delta \rho_{u}^{p s}-\mu_{, i} i_{u, i}^{p s}-d t^{p s}+\lambda_{, i} z_{u, i}^{p s}-\lambda_{, i} \delta_{, i}^{p s} \\
& \Delta p_{u, i}^{p s}=\Delta \rho_{u}^{p s}+\mu_{, i} i_{u, i}^{p s}-d t^{p s}-d_{, i}^{p s}
\end{aligned}
$$

$\Delta \phi_{u, i}^{p s}$ and $\Delta p_{u, i}^{p s}$ represents the single differenced carrierphase and pseudorange measurements. $\Delta \rho_{u}^{p s}$ is the geometric range between single difference satellites and user position and tropospheric delay. $t_{u}^{p s}$ is the first order slant ionospheric delay and $\mu_{i}$ is the frequency dependent co-efficient $\left(\mu_{i}=\frac{f_{1}^{2}}{f_{i}^{2}}\right) . d t^{p s}$ is the single differenced satellite clock and $d_{, i}^{p s}$ is the single differenced pseudorange equipment delay. $z_{u, i}^{p s}$ is the single differenced 
ambiguity and $\delta_{. i}^{p s}$ is the single differenced carrier-phase equipment delay which are expressed in cycles and scaled by the wavelength $\lambda_{, i}$.

The user observation Eqs. (1 and 2) do not contain sufficient information to solve for an integer ambiguity resolved user position. Ambiguity resolution would become possible if information about the satellite clocks and equipment delays were provided to the user. Using such externally provided information $\left(d t^{p s}, \delta^{p s}, d^{p s}\right)$ to correct the observations as

$$
\begin{aligned}
& \Delta \phi_{u, i}^{\prime p s}=\Delta \phi_{u, i}^{p s}+d t^{p s}+\lambda_{, i} \delta_{, i}^{p s} \\
& \Delta p_{u}^{\prime p s}=\Delta p_{u, i}^{p s}+d t^{p s}+d_{, i}^{p s}
\end{aligned}
$$

results in user-equations that take the form

$$
\begin{aligned}
& \Delta \phi_{u}^{p s s}=\Delta \rho_{u, i}^{p s}-\mu_{, i} i_{u, i}^{p s}+z_{u, i}^{p s} \\
& \Delta p_{u}^{p s}=\Delta \rho_{u, i}^{p s}+\mu_{, i} i_{u, i}^{p s}
\end{aligned}
$$

By utilizing externally provided corrections the user's system of observation Eqs. (5 and 6) can be solved (Teunissen and Khodabandeh 2015).

Presented in the following section is an overview of the different public providers of products that enable PPP-AR, the products and how they are applied to the PPP user equations.

\section{Public PPP-AR products}

Currently, there are three main public providers of products that enable PPP-AR. These include School of Geodesy and Geomatics at Wuhan University (SGG-WHU) (Li et al. 2015; Wuhan University 2017) which provides global post processed FCB products, Natural Resources Canada (Collins 2008; NRCan 2015) which provides post-processed DC products and Centre national d'études spatiales (Laurichesse et al. 2009; CNES 2015) which provides post-processed and real-time IRC products.

\section{Fractional cycle bias (FCB) model}

The initial application of ambiguity resolution to PPP was made by Ge et al. (2008) using the FCB model. The FCB method estimates the equipment delay by averaging the fractional parts of the steady-state float ambiguity estimates (Ge et al. 2008) to be removed from common satellite clock estimates. Presented in Eqs. $(7,8,9)$ is the application of the FCB products $d \tilde{t}^{p s}, \tilde{a}_{q, 1}^{p s}$ and $\tilde{a}_{q, W}^{p s}$ where $\sim$ represents the products that were estimated from within the network solution and $\approx$ represents the corrected user equations. IF represents the ionospheric-free linear combination and WN represents the MelbourneWübbena combination.

$$
\begin{aligned}
& \Delta \tilde{\tilde{\phi}}_{u, I F}^{p s}=\Delta \phi_{u, I F}^{p s}+d \tilde{t}^{p s}-\lambda_{N} \tilde{a}_{q, 1}^{p s}-\frac{\lambda_{2}}{\mu_{12}} \tilde{a}_{q, W N}^{p s} \\
& \Delta \tilde{\tilde{p}}_{u, I F}^{p s}=\Delta p_{u, I F}^{p s}+d \tilde{t}^{p s} \\
& \Delta \widetilde{\tilde{\phi}}_{u, W N}^{p s}=\Delta \phi_{u, W N}^{p s}-\lambda_{W} \tilde{a}_{q, W}^{p s}
\end{aligned}
$$

Currently SGG-WHU produces 14 sets of FCB products corresponding to different precise products such as those from COD, ESA, GFZ, GRGS, IGR, and IGS which are estimated daily ( $\mathrm{Li}$ et al. 2015). The FCB products can be downloaded from, ftp://gnss.sgg.whu.edu.cn/product/FCB and have the prefix "SGG" and the naming convention includes the AC used for generating the FCBs. FCBs generated using the final IGS products were selected as this set of FCB products capitalized on the accuracy and reliability of the combined products IGS.

Presented in Fig. 1 is the relative satellite clock error, $d \tilde{t}^{p s}$ for PRN 10 with respect to satellite PRN 27. Relative satellite clock is presented to eliminate the time scale factor because of the reference clock selected by the Analysis Center.

Presented in Fig. 2 is what is referred to as "narrow lane" $\tilde{a}_{q, 1}^{p s}$ and the wide lane FCB correction $\tilde{a}_{q, W N}^{p s}$ for PRN 10. The products are transmitted at a 15-min data rate. The wide lane FCBs $\tilde{a}_{q, W}^{p s}$ are transmitted once every $24 \mathrm{~h}$. Geng (2010) describes the wide lane FCBs are very stable over several days, or even a few months.

\section{Decoupled clock (DC) model}

The underlying concept of the DC model presented by Collins et al. (2008) is the carrier-phase and pseudorange measurements are not synchronized with each other at equivalent level of precision. The timing of the different observables must be considered separately, if they are to be processed together rigorously. The DC model is a reformulation of the ionosphere-free carrier-phase and pseudorange observation equations presented in Eqs (10)

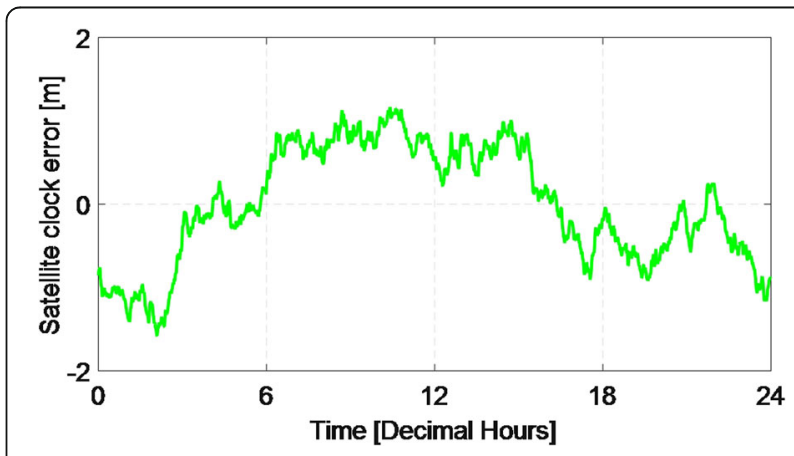

Fig. 1 Relative satellite clock correction provided by Wuhan University on DOY 28 of 2015 for PRN 10 (relative to PRN 6 and 27). Linear trend has been removed. All units are in metres 


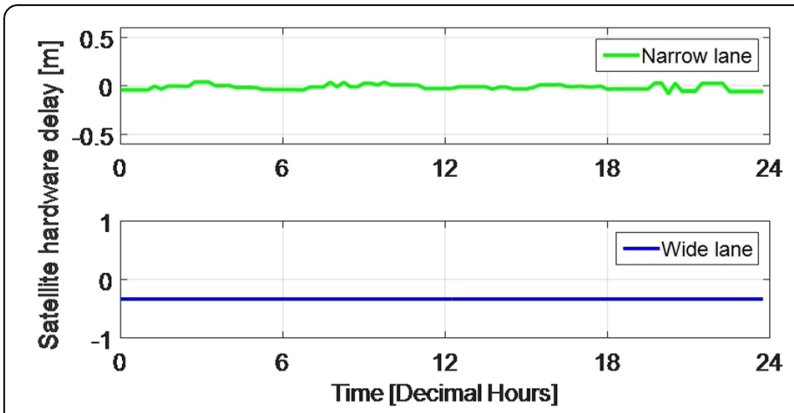

Fig. 2 Narrow lane (upper subplot) and wide lane (lower subplot) FCB provided by Wuhan University on DOY 28 of 2015 for PRN 10. All units are in metres

and (11). When combined with the narrow lane pseudorange, Eq. (12) and the wide lane phase, Eq. (13) allows for ambiguity resolution. The DC products transmitted to the user are $\delta \tilde{t}_{I F}^{p s}, d \tilde{t}^{p s}$ and $\tilde{\delta}_{W N}^{p s}$.

$$
\begin{aligned}
\Delta \tilde{\tilde{\phi}}_{u, I F}^{p s} & =\Delta \phi_{u, I F}^{p s}+\delta \tilde{t}_{I F}^{p s} \\
\Delta \widetilde{\tilde{p}}_{u, I F}^{p s} & =\Delta p_{u, I F}^{p s}+d \tilde{t}^{p s} \\
\Delta \tilde{\tilde{p}}_{u, N L}^{p s} & =\Delta p_{u, N L}^{p s}+\delta \tilde{t}_{I F}^{p s}+\lambda_{W L} \tilde{\delta}_{W N}^{p s} \\
\Delta \widetilde{\tilde{\phi}}_{u, W L}^{p s} & =\Delta \phi_{u, W L}^{p s}+\delta \tilde{t}_{I F}^{p s}
\end{aligned}
$$

Where $N L$ represents the narrow lane linear combination and $W L$ represents the wide lane linear combination. The reformulated DC model using $\Delta \widetilde{\tilde{p}}_{u, N L}^{p s}$ and $\Delta \widetilde{\widetilde{\phi}}_{u, W L}^{p s}$ rather than $\Delta \widetilde{\tilde{\phi}}_{u, W N}^{p s}$ was carried out to allow the PPP user to utilize the estimation of the slant ionospheric term for instantaneous re-convergence. (Collins and Bisnath 2011).

Presented in Fig. 3 is the relative satellite carrier-phase clock error, $\delta \tilde{t}_{I F}^{p s}$ for PRN 10 with respect to satellite PRN 27.

Figure 4 illustrates the relative satellite pseudorange clock error, $\left(d \tilde{t}^{p s}-\delta \tilde{t}_{I F}^{p s}\right)$ and the relative wide lane clock

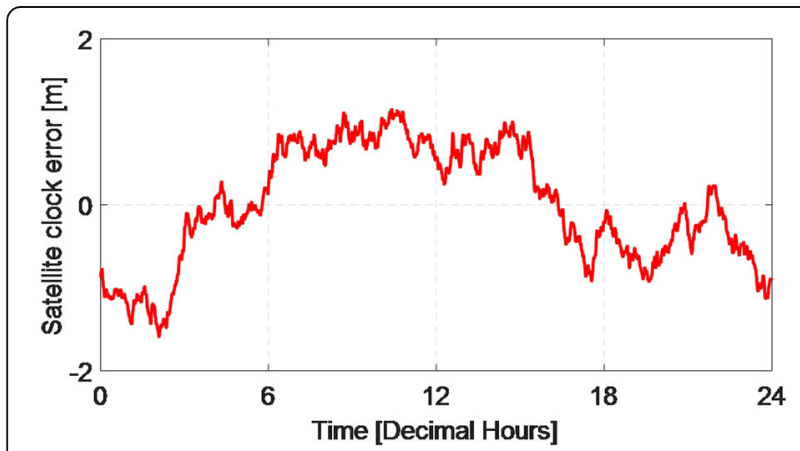

Fig. 3 Relative satellite carrier-phase clock correction provided by NRCan on DOY 28 of 2015 for PRN 10 (relative to PRN 27). Linear trend has been removed. All units are in metres
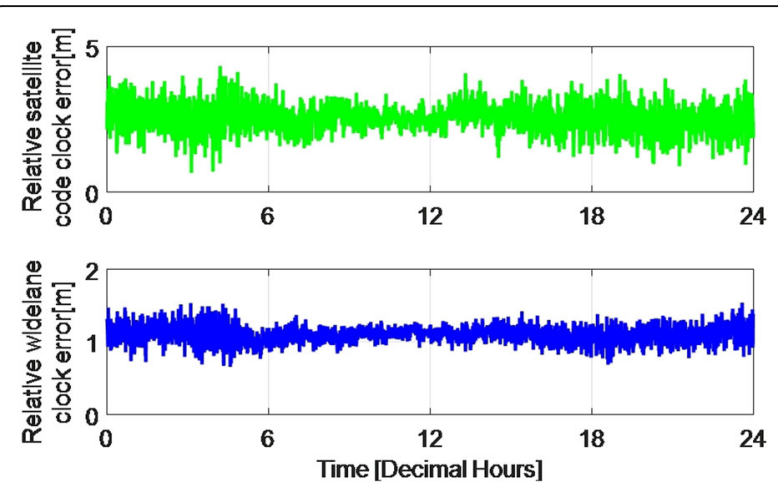

Fig. 4 Relative code clock error (upper figure) and wide lane (lower figure) DC provided by NRCan on DOY 28 of 2015 for PRN 10. All units are in metres

error, $\left(\tilde{\delta}_{W N}^{p s}-\delta \tilde{t}_{I F}^{p s}\right)$ for PRN 10. The term "relative" is used because they are with respect to the $\delta \tilde{t}_{I F}^{p s}$, similar to the other providers, even though their naming convention gives the reader the impression that they absolute correction terms. The relative satellite pseudorange and wide lane clock errors are nosier because it is unfiltered, in contrast to the FCB and IRC products. All products are transmitted at a $30 \mathrm{~s}$ data rate.

\section{Integer recovery clock (IRC) model}

The IRC model was presented by Mercier and Laurichesse (2007) and Laurichesse and Mercier (2007). The products by CNES, consists of wide lane satellite equipment delays and the carrier-phase satellite clocks. The wide lane satellite equipment delays are daily wide lane pseudorange/carrierphase equipment delays by averaging arc-dependent estimates using the Melbourne-Wübbena combination. The carrier-phase satellite clocks which are aligned to the satellite pseudorange clocks within a narrow lane cycle. The alignment of the carrier-phase clocks allows the clocks to be used for the pseudorange and carrierphase measurements. Presented in Eqs. $(14,15,16,17)$ is the application of the IRC products $\delta \tilde{t}_{I F}^{p s}$ and $\tilde{\delta}_{W N}^{p s}$.

$$
\begin{aligned}
\Delta \widetilde{\tilde{\phi}}_{u, I F}^{p s} & =\Delta \phi_{u, I F}^{p s}+\delta \tilde{t}_{I F}^{p s} \\
\Delta \tilde{\tilde{p}}_{u, I F}^{p s} & =\Delta p_{u, I F}^{p s}+\delta \tilde{t}_{I F}^{p s} \\
\Delta \widetilde{\tilde{p}}_{u, N L}^{p s} & =\Delta p_{u, N L}^{p s}+\delta \tilde{t}_{I F}^{p s}+\lambda_{W L} \tilde{\delta}_{W N}^{p s} \\
\Delta \tilde{\tilde{\phi}}_{u, W L}^{p s} & =\Delta \phi_{u, W L}^{p s}+\delta \tilde{t}_{I F}^{p s}
\end{aligned}
$$

The IRC products can be downloaded from, https://igsaccnes.cls.fr/html/products.html and have the prefix "GRG". From this point, onwards, these products shall be referred to as IRC (GRG). 
In 2014, the IRC model was reformulated and adopted an uncombined representation of their products, as such, satellite equipment delays are provided for each observable $\left(\tilde{\delta}_{, i}^{p s}, \tilde{d}_{, i}^{p s}\right.$ and share a common satellite clock $\left(\tilde{d}_{, i}^{p s}\right)$ (Laurichesse 2014).

$$
\begin{aligned}
\Delta \tilde{\tilde{\phi}}_{u, i}^{p s} & =\Delta \phi_{u, i}^{p s}+d \tilde{t}^{p s}+\lambda_{, i} \tilde{\delta}_{, i}^{p s} \\
\Delta \tilde{\tilde{p}}_{u, i}^{p s} & =\Delta p_{u, I F}^{p s}+d \tilde{t}^{p s}+\tilde{d}_{, i}^{p s}
\end{aligned}
$$

The products can be downloaded from, http://www.pppwizard.net/products/ and have the prefix "CNT". From this point, onwards, these products shall be referred to as IRC (CNT). Presented in Fig. 5 is the relative satellite clock error, $d \tilde{t}^{p s}$ for PRN 10 with respect to satellite PRN 27.

Presented in Fig. 6 are the observable dependent satellite equipment delays for the pseudorange $\left(\tilde{d}_{. i}^{p s}\right)$ and carrierphase measurements $\left(\tilde{\delta}_{, i}^{p s}\right) . \tilde{d}_{, i}^{p s}$ is modelled as a constant term $\tilde{d}_{i,}^{p s} . \tilde{d}_{, i}^{p s}$ are assumed constant over a 24 -h period and $\tilde{\delta}_{, i}^{p s}$ is modelled as unconstrained. All products are transmitted at a $5 \mathrm{~s}$ data rate and available in the SINEX BIAS format (Schaer 2016).

\section{Summary}

The user implementation examines the three public providers of products to enable PPP-AR are listed in Table 1. The criteria include the different products transmitted, data rate and different assumptions made.

\section{Product transformation}

While the different strategies (FCB, DC, IRC) make different assumptions, there are fundamental similarities between them. For the PPP user, the mathematical model is similar; the different PPP-AR products contain the same information and as a result should permit one-to-one transformations between them, allowing interoperability of the PPP-AR products. The advantage of interoperability of the different

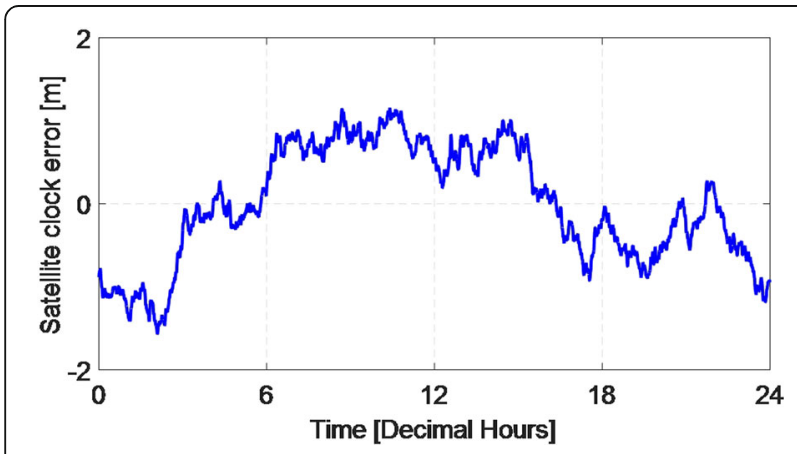

Fig. 5 Relative satellite clock correction provided by CNES on DOY 28 of 2015 for PRN 10 (relative to PRN 27). Linear trend has been removed. All units are in metres

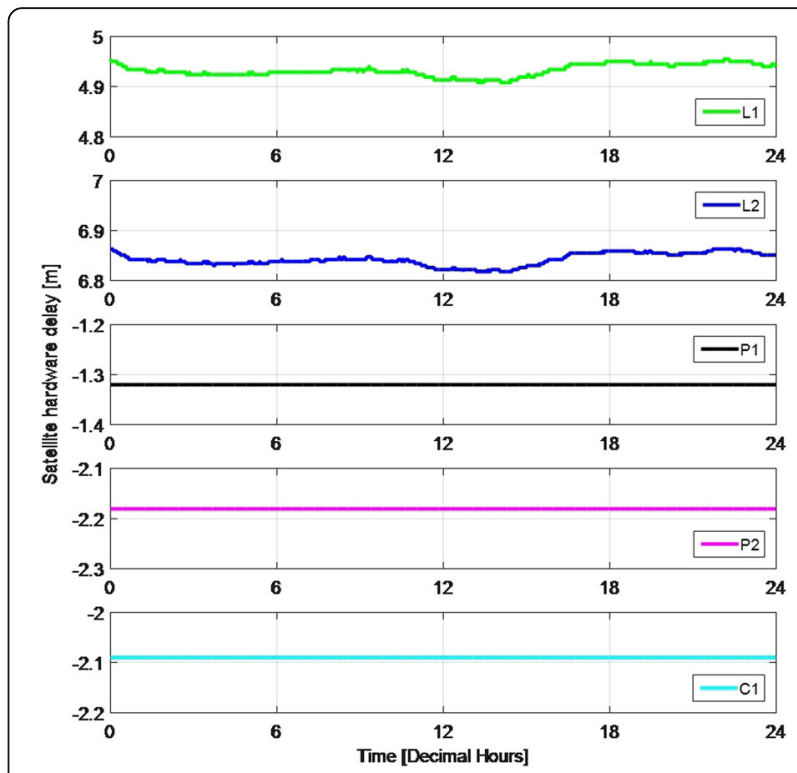

Fig. 6 Observable dependent satellite equipment delays for PRN 10 IRC provided by CNES on DOY 28 of 2015 for PRN 10

PPP-AR products would be to permit the PPP user to transform independently generated PPP-AR products to obtain multiple fixed solutions of comparable precision and accuracy. The ability to provide multiple solutions would increase the reliability of the solution for, e.g., real-time processing; if there was an outage in the generation of the PPP-AR products, the user can instantly switch streams to a different provider. The following sections examine the transformation matrix used to transform the IRC and FCB products to the DC format. While the original DC format are in units of seconds and nanoseconds, the following transformed parameters are in units of meters.

\section{Fractional cycle bias}

The FCB products consist of $d \tilde{t}_{I F}^{p s}, \tilde{a}_{q, 1}^{p s}$ and $\tilde{a}_{q, W N}^{p s}$ which has been estimated from the network solutions. The FCBs utilized within this analysis were generated using IGS final orbit and clock products. The fundamental differences between the FCB and DC is that $\tilde{a}_{q, 1}^{p s}$ was not determined in the DC method, but assimilated within the clock estimates. Also, $\tilde{a}_{q, W}^{p s}$ are assumed constant over a 24-h period whereas in the DC method the $\tilde{\delta}_{W N}^{p s}$ is neither constrained nor smoothed. The difference in symbology between the FCB and DC approaches is a result of the different representation of the products. DC products are described as a clock term, whereas the FCB products are described as a correction term to be applied to the ambiguity.

Presented in Eq. (20) is a simplified transformation matrix to transform from FCB to DC model. The original transformation model was presented in Teunissen and 
Table 1 Comparison of different public providers of products to enable PPP-AR

\begin{tabular}{|c|c|c|c|c|c|c|c|c|}
\hline \multirow{4}{*}{ PPP-AR Products } & \multirow{2}{*}{\multicolumn{2}{|c|}{$\begin{array}{l}\text { Fractional Cycle } \\
\text { Bias (FCB) model }\end{array}$}} & \multirow{2}{*}{\multicolumn{2}{|c|}{$\begin{array}{l}\text { Decoupled Clock } \\
\text { (DC) model }\end{array}$}} & \multicolumn{4}{|c|}{ Integer Recovery Clock (IRC) model } \\
\hline & & & & & \multicolumn{2}{|c|}{ Combined (GRG) } & \multicolumn{2}{|c|}{ Uncombined (CNT) } \\
\hline & \multirow{2}{*}{$\begin{array}{l}d \tilde{t}_{l F}^{p s} \\
\tilde{a}_{q, W N}^{p s} \\
\tilde{a}_{q, 1}^{p s}\end{array}$} & \multirow{2}{*}{$\begin{array}{l}\text { - code clock } \\
\text { - wide lane } \\
\text { - narrow lane }\end{array}$} & \multirow{2}{*}{$\begin{array}{l}\delta \tilde{t}_{I F}^{p s} \\
d \tilde{t}_{I F}^{p s} \\
\tilde{\delta}_{W N}^{p s}\end{array}$} & \multirow{2}{*}{$\begin{array}{l}\text { - phase clock } \\
\text { - code clock } \\
\text { - wide lane } \\
\text { clock }\end{array}$} & \multirow{2}{*}{$\begin{array}{l}\delta \tilde{t}_{\mathbb{F}}^{p s} \\
\tilde{\delta}_{W N}^{p s}\end{array}$} & \multirow{2}{*}{$\begin{array}{l}\text { - phase clock } \\
\text { - wide lane clock }\end{array}$} & \multirow{2}{*}{$\begin{array}{l}d \tilde{t}_{1 F}^{p s} \\
\tilde{\delta}_{, i}^{p s} \\
\tilde{d}_{, i}^{p s}\end{array}$} & \multirow{2}{*}{$\begin{array}{l}\text { - code clock } \\
\text { - phase equipment delay } \\
\text { - code equipment delay }\end{array}$} \\
\hline & & & & & & & & \\
\hline $\begin{array}{l}\text { Provided product } \\
\text { units }\end{array}$ & $\begin{array}{l}d \tilde{t}_{\text {IF }}^{p s} \\
\tilde{a}_{q, W N}^{p s} \\
\tilde{a}_{q, 1}^{p s}\end{array}$ & $\begin{array}{l}\text { - seconds } \\
\text { - cycles } \\
\text { - cycles }\end{array}$ & $\begin{array}{l}\delta \tilde{t}_{I F}^{p s} \\
\delta \tilde{t}_{I F}^{p s}-d \tilde{t}_{I F}^{p s} \\
\tilde{\delta}_{W N}^{p s}\end{array}$ & $\begin{array}{l}\text { - seconds } \\
\text { - nanoseconds } \\
\text { - nanoseconds }\end{array}$ & $\begin{array}{l}\delta \tilde{t}_{\mathbb{F}}^{p s} \\
\tilde{\delta}_{W N}^{p s}\end{array}$ & $\begin{array}{l}\text { - seconds } \\
\text { - cycles }\end{array}$ & $\begin{array}{l}d \tilde{t}^{p s} \\
\tilde{\tilde{\delta}}_{\tilde{p}}^{p \xi} \\
\tilde{d}_{, i}^{p s}\end{array}$ & $\begin{array}{l}\text { - seconds } \\
\text { - nanoseconds } \\
\text { - nanoseconds }\end{array}$ \\
\hline \multirow[t]{2}{*}{ Data rate } & $d \tilde{t}^{p s}$ & $-30 s$ & $\delta \tilde{t}_{\mathbb{F}}^{p s}$ & $-30 s$ & $\delta \tilde{t}_{\mathbb{F}}^{p s}$ & $-30 s$ & \multirow{2}{*}{\multicolumn{2}{|c|}{$\begin{array}{ll}\tilde{d}_{\tilde{d}^{p s}}^{p s} \tilde{\delta}_{, i}^{p s} & -5 s \\
& -5 s \\
& -5 s\end{array}$}} \\
\hline & $\begin{array}{l}\tilde{a}_{q, 1}^{p s} \\
\tilde{a}_{q, W N}^{p s}\end{array}$ & $\begin{array}{l}\text { - } 15 \text { min } \\
\text { - daily }\end{array}$ & $\begin{array}{l}d \tilde{t}^{p s} \\
\tilde{\delta}_{W N}^{p s}\end{array}$ & $\begin{array}{l}-30 s \\
-30 s\end{array}$ & $\tilde{\delta}_{W N}^{p s}$ & - daily & & \\
\hline General assumptions & \multicolumn{2}{|c|}{$\begin{array}{l}\text { Constant } \tilde{a}_{q, W}^{p s} \text { are } \\
\text { estimated daily by } \\
\text { averaging arc-dependent } \\
\text { estimates. }\end{array}$} & \multicolumn{2}{|c|}{$\begin{array}{l}\text { No constraints or } \\
\text { smoothing applied. }\end{array}$} & \multicolumn{2}{|c|}{$\begin{array}{l}\delta \tilde{t}_{I F}^{p s} \text { aligned to the satellite } \\
\text { pseudorange clocks within a } \\
\text { narrow lane cycle. } \tilde{\delta}_{W N}^{p s} \text { represents } \\
\text { a daily average }\end{array}$} & \multicolumn{2}{|c|}{$\begin{array}{l}\tilde{\delta}_{W N}^{p s} \text { estimated as unconstrained in } \\
\text { the network work solution with } \\
\text { white noise added at each epoch. }\end{array}$} \\
\hline Products used & \multicolumn{2}{|c|}{ Post-processed } & \multicolumn{2}{|c|}{ Post-processed } & \multicolumn{2}{|c|}{ Post-processed } & \multicolumn{2}{|c|}{ Archived real-time } \\
\hline Network solution & \multicolumn{2}{|c|}{$\begin{array}{l}\text { Global. } \\
\text { IGS final products }\end{array}$} & \multicolumn{2}{|c|}{$\begin{array}{l}\text { Global. } \\
\text { EMR rapid products }\end{array}$} & \multicolumn{2}{|c|}{$\begin{array}{l}\text { Global. } \\
\text { GRG final products }\end{array}$} & \multicolumn{2}{|c|}{$\begin{array}{l}\text { Global. } \\
\text { GRG ultra-rapid products }\end{array}$} \\
\hline PPP user model & \multirow{3}{*}{\multicolumn{4}{|c|}{$\Delta p_{u, \mid F}^{p s}, \Delta \phi_{u, F}^{p s}, \Delta p_{u, N L}^{p s}, \Delta \phi_{u, W L}^{p s}$ or $\Delta p_{u, \mid F}^{p s}, \Delta \phi_{u, F}^{p s}, \Delta \phi_{u, W N}^{p s}$}} & & & \multirow{3}{*}{\multicolumn{2}{|c|}{$\begin{array}{l}\text { User defined } \\
\tilde{d}_{, i}^{p s}\end{array}$}} \\
\hline P1, P2 correction & & & & & & & & \\
\hline$L 1, L 2$ correction & & & & & & & & \\
\hline NL correction & \multicolumn{4}{|l|}{$\tilde{a}_{q, 1}^{p s}$} & & & & \\
\hline WL correction & \multicolumn{2}{|l|}{$\begin{array}{l}q, 1 \\
\tilde{a}_{q, W N}^{p s}\end{array}$} & \multicolumn{2}{|l|}{$\tilde{\delta}_{W N}^{p s}$} & \multicolumn{4}{|l|}{$\tilde{\delta}_{W N}^{p s}$} \\
\hline
\end{tabular}

Khodabandeh (2015) included the terms $z_{, 1}^{p s}$ and $z_{, W N}^{p s}$ which represented an ambiguous integer offset. While formally required, in practice the arbitrary integer natured offset is absorbed by the ambiguity term.

$$
\left[\begin{array}{l}
d \tilde{t}_{I F}^{p s} \\
\delta \tilde{t}_{I F}^{p s} \\
\tilde{\delta}_{W N}^{p s}
\end{array}\right]=\left[\begin{array}{ccc}
1 & 0 & 0 \\
1 & \lambda_{N L} & 0 \\
0 & 0 & \lambda_{W N}
\end{array}\right]\left[\begin{array}{c}
d \tilde{t}_{I F}^{p s} \\
\tilde{a}_{, 1}^{p s} \\
\tilde{a}_{, W N}^{p s}
\end{array}\right]
$$

where, on the RHS, $d \tilde{t}_{I F}^{p s}$ is in units of meters $\tilde{a}_{q, 1}^{p s}$ and $\tilde{a}_{q, W N}^{p s}$ are in units of cycles.

\section{Integer recovery clock}

The IRC (GRG) method is similar to the DC model, such that, the carrier-phase satellite equipment delays were assimilated within the carrier-phase satellite clocks. As such, utilizing the products within the software does not require any modification of the products. IRC and DC adopted similar philosophies where the PPP-AR products are considered as a satellite clock correction term. The primary difference is the alignment of the carrier phase clocks and the filtering applied.

$$
\left[\begin{array}{l}
d \tilde{t}_{I F}^{p s} \\
\delta \tilde{t}_{I F}^{p s} \\
\tilde{\delta}_{W N}^{p s}
\end{array}\right]=\left[\begin{array}{cc}
1 & 0 \\
1 & 0 \\
0 & \lambda_{W N}
\end{array}\right]\left[\begin{array}{l}
\delta \tilde{t}_{I F}^{p s} \\
\tilde{\delta}_{W N}^{p s}
\end{array}\right]
$$

where, on the RHS, $\delta \tilde{t}_{I F}^{p s}$ is in units of meters and $\tilde{\delta}_{W N}^{p s}$ is in units of cycles.

The IRC (CNT) products are uncombined, such that, one satellite equipment delay per observable is identified and broadcasted. The primary benefit of such an approach is the interoperability, allowing the network side and user side to implement different ambiguity resolution strategies. Equation (22) is the transformation of the independent clocks to the Radio Technical Commission for Maritime (RTCM) state space representation (SSR) standard to enable PPP-AR. The representation consists of a common clock and one satellite equipment delay per observable (Laurichesse 2014).

$$
\left[\begin{array}{c}
d \tilde{t}_{I F}^{p s} \\
\delta \tilde{t}_{I F}^{p s} \\
\tilde{\delta}_{W N}^{p s}
\end{array}\right]=\left[\begin{array}{ccccc}
1 & \frac{\mu_{2}}{\mu_{12}} & -\frac{\mu_{1}}{\mu_{12}} & 0 & 0 \\
1 & 0 & 0 & \frac{\mu_{2}}{\mu_{12}} & -\frac{\mu_{1}}{\mu_{12}} \\
0 & \kappa \frac{\lambda_{W L}}{\lambda_{1}} & \kappa \frac{\lambda_{W L}}{\lambda_{2}} & \frac{\lambda_{W L}}{\lambda_{1}} & -\frac{\lambda_{W L}}{\lambda_{2}}
\end{array}\right]\left[\begin{array}{c}
d \tilde{t}_{I F}^{p s} \\
\tilde{d}_{, 1}^{p s} \\
\tilde{d}_{, 2}^{p s} \\
\tilde{\delta}_{, 1}^{p s} \\
\tilde{\delta}_{, 2}^{p s}
\end{array}\right]
$$


Where $\kappa$ represents $\frac{\lambda_{1}-\lambda_{2}}{\lambda_{1}+\lambda_{2}}$ and on the RHS $d \tilde{t}_{I F}^{p s}, \tilde{d}_{, 1}^{p s}$ and $\tilde{d}_{, 2}^{p s}$ are in units of meters and $\tilde{\delta}_{, 1}^{p s}$ and $\tilde{\delta}_{, 2}^{p s}$ are in units of cycles.

\section{Analysis of transformed products}

While the transformations in the previous section assumed the transformed satellite clocks and equipment delays were in units of meters, the results in this section are presented in terms of time, as the satellite equipment delays should be thought of as an unmodelled timing error. For comparison of the products, the transformed products were corrected for the orbital radial error as the satellite clock and orbital radial error are strongly correlated.

Figure 7 illustrates the transformed FCB, IRC (GRG) and IRC $(\mathrm{CNT})$ to carrier-phase satellite clocks. The FCB and IRC (CNT) are common clocks which required the inclusion of the satellite carrier-phase equipment delays. FCB, IRC and DC satellite carrier-phase satellite clocks closely agree with a difference of relative products being less than $0.07 \mathrm{~ns}$.

Presented in Fig. 8 is the relative satellite clock error $\left(d \tilde{t}_{I F}^{p s}-\delta \tilde{t}_{I F}^{p s}\right)$ for the transformed FCB, IRC (GRG) and IRC (CNT) products. For the original DC product, a simple moving average filter was applied with a bin size of $5 \mathrm{~min}$, to reduce the noise and illustrate the underlying satellite equipment delay. The relative satellite clock error represents the difference between the pseudorange and carrier-phase clocks. The distinct differences of the products are easily visible, such as the filtering present within the FCB and IRC products in contrast to the DC. As previously mentioned, relative satellite clock error for the IRC (GRG) products are assumed to be zero because satellite carrier phase and pseudorange clocks are aligned within a narrow lane cycle. The philosophy of the DC products is, the pseudorange measurements are naturally noisier than the carrier-phase measurements and should

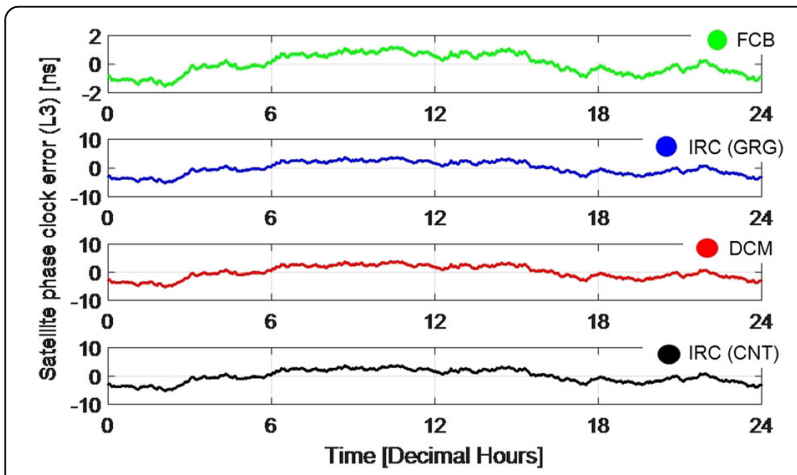

Fig. 7 Transformed FCB, IRC (GRG) and IRC (CNT) satellite phase clock correction on DOY 28 of 2015 for PRN 10 (relative to PRN 27). DC was included for comparison. Linear trend has been removed. All units are in nanoseconds

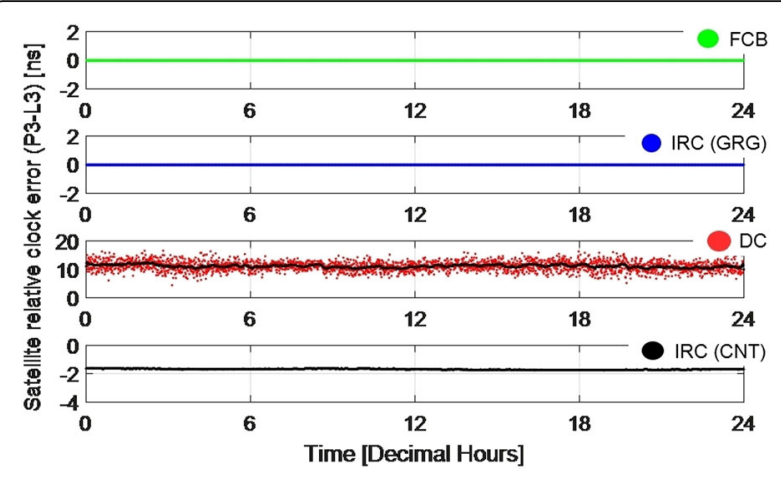

Fig. 8 Transformed FCB, IRC (GRG) and IRC (CNT) to code-phase relative clock correction on DOY 28 of 2015 for PRN 10 (relative to PRN 27). DC was included for comparison. All units are in nanoseconds

be presented as such, allowing the PPP user the flexibility to filter the satellite pseudorange equipment delays if required. These terms are expected to have different offsets present within them because of different timing constraints imposed by the respective AC.

Presented in Fig. 9 is the relative satellite wide lane clock error for the transformed FCB, IRC (GRG) and IRC (CNT) products. Similarly, a simple moving average filter was applied DC product with a bin size of $5 \mathrm{~min}$, to reduce the noise and illustrate the underlying satellite equipment delay. The relative satellite clock error represents the difference between the wide lane clocks and carrier-phase clocks. As expected, the transformed FCB, IRC (GRG) and IRC (CNT) products had a constant wide lane estimates.

\section{Performance of transformed products}

GNSS data from 55 globally distributed stations were processed using data from DOY 25 to 31, GPS week 1829, of 2015 provided by IGS. The data were processed using the York-PPP software (Seepersad 2012; Aggrey 2015). York-PPP was developed based on the processing

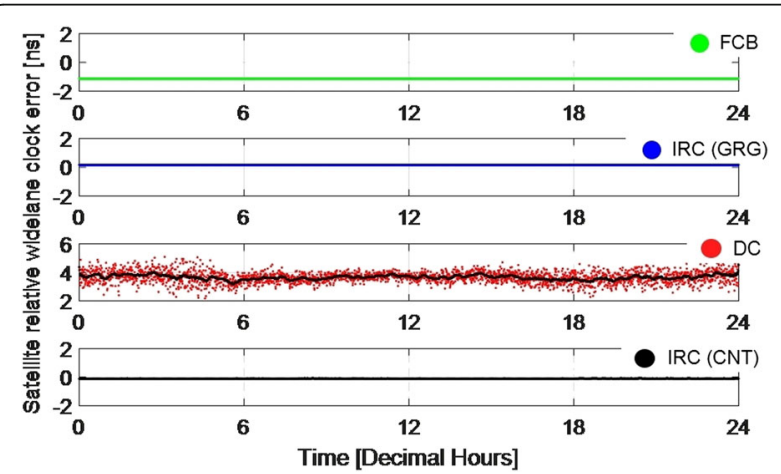

Fig. 9 Transformed FCB, IRC (GRG) and IRC (lower) to relative wide lane clock correction on DOY 28 of 2015 for PRN 10 (relative to PRN 27). DC (middle) was included for comparison. All units are in nanoseconds 
engine used by the online CSRS-PPP service (NRCan, 2015). A global distribution of the sites was selected and illustrated in Fig. 10. The linear combination of the measurements consisted of ionosphere-free pseudorange and carrier-phase, narrow lane pseudorange and wide lane carrier-phase.

The station was analyzed in static mode. Receiver clocks were estimated epoch-by-epoch. The zenith tropospheric delays were also estimated each epoch with a random walk co-efficient of $2 \mathrm{~cm} / \mathrm{sqrt}$ (hour). The station coordinates were initialized using a pseudorange only solution with an initial constraint of $10 \mathrm{~m}$. The IGS absolute antenna model file was used and ocean loading coefficients were obtained from Scherneck (2013) for each of the sites processed. An elevation cut-off angle was set to $10^{\circ}$. The transformed IRC (GRG) and IRC (CNT) products were processed with their original satellite orbit files to maintain consistency between satellite orbits, clocks and equipment delays.

Presented in Table 2 is the rms of the final solution of 24-h datasets using data from 55 IGS sites utilizing the satellite products provided by NRCan, CNES, Wuhan University and IGS. Ambiguity resolution was performed at each epoch utilizing satellites with an elevation angle greater than $25^{\circ}$. Equivalent performance was noted utilizing the DC, IRC (GRG) and FCB products. Of the four solutions, FCB products had the highest accuracy. This is attributed to the products being generated using final IGS orbit and clock products. To confirm this, FCBs generated using GRG orbit and clock products were also examined and comparable performance was observed. The least accurate solution was obtained using the IRC $(\mathrm{CNT})$ products, which is due to the products being archived real time products.

The GNSS site located in Algonquin, Canada (ALGO) on DOY 28 of 2015 was selected because similar performance was observed within the week of processing and it illustrated similar trends to other GNSS sites examined. Presented in Fig. $11(\mathrm{a}-\mathrm{d})$ is the horizontal component and Fig. $11(\mathrm{e}-\mathrm{h})$ the vertical component for the DC product and the transformed IRC (GRG), IRC (CNT) and FCB products. The subplots within Fig. 11 illustrates both the "float" and "fixed" solution, where fixed represents the ambiguity resolved solution and float the unresolved solution. A stringent convergence threshold of $5 \mathrm{~cm}$ was set to examine the time the solution took to converge.

The horizontal position error, Fig. $11(\mathrm{a}-\mathrm{d})$, all four solutions had an overshoot of 55-60 cm after $30 \mathrm{~s}$ of data processing. Float and fixed solutions were equivalent within the first $10 \mathrm{~min}$ as fixing was only performed after $10 \mathrm{~min}$. Convergence of the float solution to the predefined threshold $(5 \mathrm{~cm})$ was $14 \mathrm{~min}$ and fixing the ambiguities improved convergence by only $1 \mathrm{~min}$. Similar convergence was noted in the horizontal component for all four solutions.

The strength of applying ambiguity resolution was illustrated in the time to attain a steady state. All three solutions illustrated different convergence trends before attaining a steady state: DC products took $5.2 \mathrm{~h}$, IRC (GRG) products took $3 \mathrm{~h}$ and IRC (CNT) as well as FCB products took $2 \mathrm{~h}$. The ambiguity resolved solution improved the time attain a steady state as the time was reduced to $1.2 \mathrm{~h}$.

The vertical position error, Fig. $11(\mathrm{e}-\mathrm{h})$, had an overshoot of 36, 45, 54 and $60 \mathrm{~cm}$ for the DC, IRC (GRG), IRC (CNT) and FCB products respectively. The same predefined threshold for the vertical component was maintained. Convergence times were 18, 16, 15 and $13 \mathrm{~min}$ for the DC, IRC (GRG), IRC (CNT) and FCB products, respectively. Applying ambiguity resolution did not improve the time to attain a steady state in the vertical component: 4.5, 3,5.8

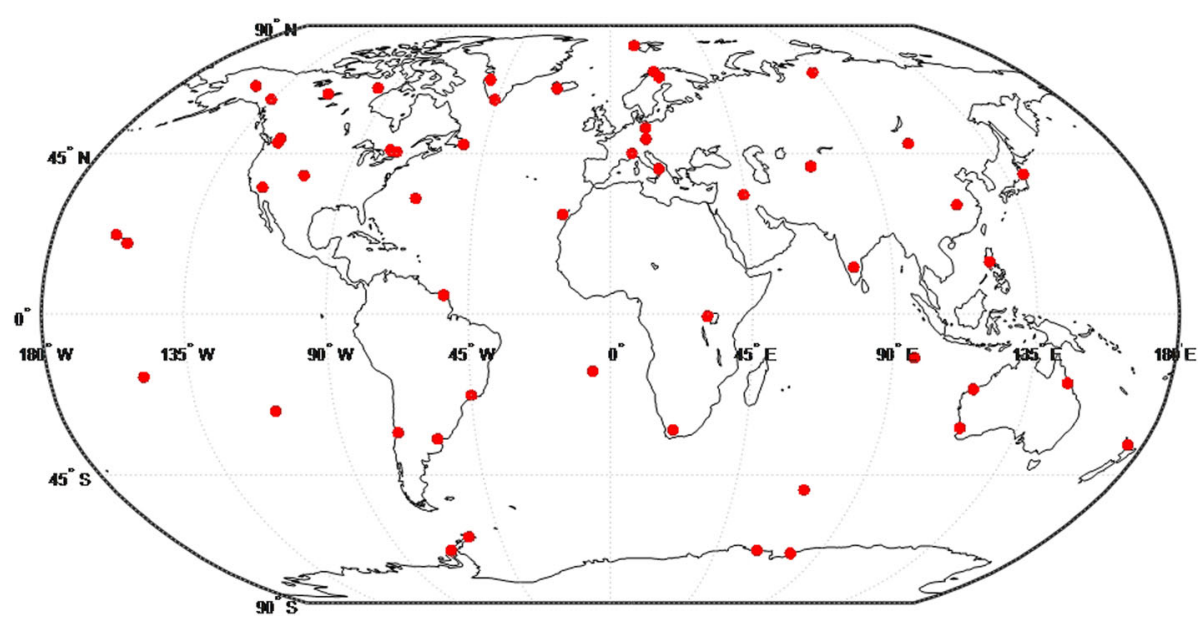

Fig. 10 Distribution of the selected 55 globally distributed IGS stations observed during DOY 25 to 31 GPS week 1829 
Table 2 rms of final solution produced by York-PPP from 24-h datasets using data from 55 sites for DOY 25 to 31, GPS week 1829, of 2015 provided by the IGS. Satellite products were provided by NRCan, CNES and Wuhan University. All units are in millimetres

\begin{tabular}{|c|c|c|c|c|c|c|c|c|}
\hline & \multicolumn{2}{|l|}{$D C$} & \multicolumn{2}{|c|}{ IRC (GRG) } & \multicolumn{2}{|c|}{ IRC (CNT) } & \multicolumn{2}{|l|}{$\mathrm{FCB}$} \\
\hline & Float & Fixed & Float & Fixed & Float & Fixed & Float & Fixed \\
\hline Northing & 5 & 5 & 5 & 4 & 7 & 6 & 5 & 4 \\
\hline Easting & 6 & 3 & 5 & 3 & 8 & 7 & 5 & 3 \\
\hline Horizontal & 8 & 6 & 7 & 5 & 11 & 9 & 7 & 5 \\
\hline Vertical & 11 & 10 & 11 & 10 & 15 & 10 & 9 & 9 \\
\hline $3 D$ & 13 & 12 & 13 & 11 & 18 & 14 & 11 & 10 \\
\hline
\end{tabular}
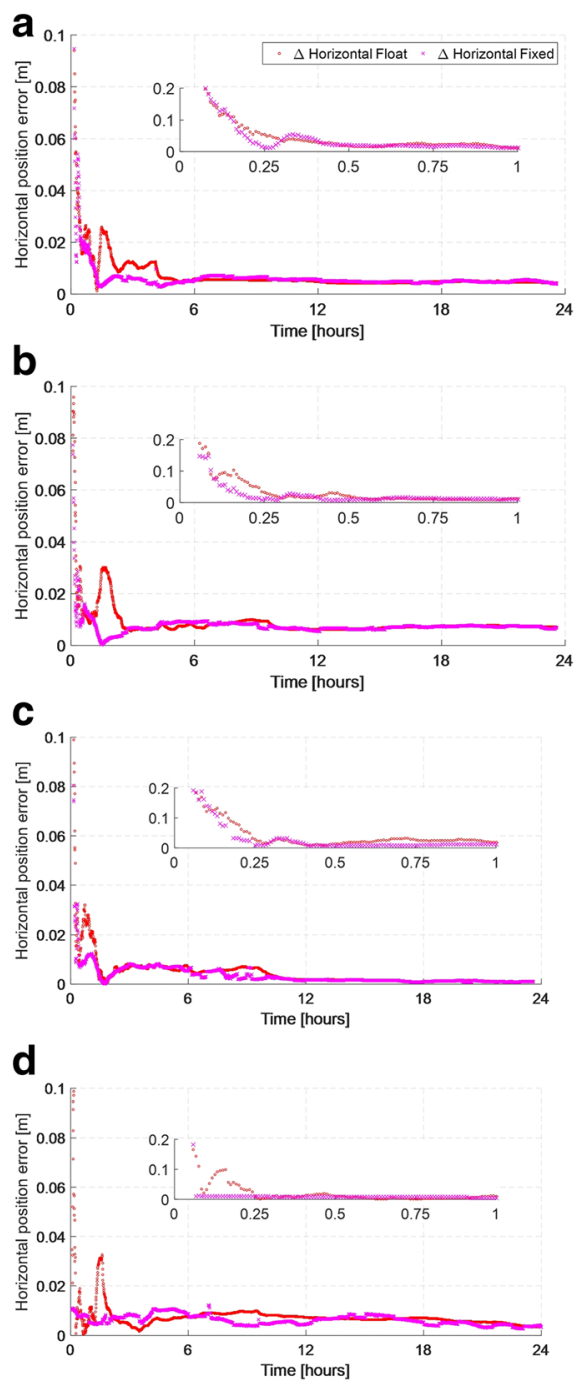

and $2 \mathrm{~h}$ for the DC, IRC (GRG), IRC (CNT) and FCB products, respectively. Slower convergence was noted in the vertical component due the strong correlation between the atmospheric effects and the vertical component. Any unmodelled components of the atmospheric effects required time averaging or a-priori information to be provided to the user (Collins and Bisnath 2011; Shi and Gao 2014).

\section{Challenges of interoperability of PPP products}

Interoperability of the different PPP products is a challenging task due to the public availability of different quality of products, limited literature documenting the conventions adopted within the network solution of the providers and unclear definitions of the corrections.
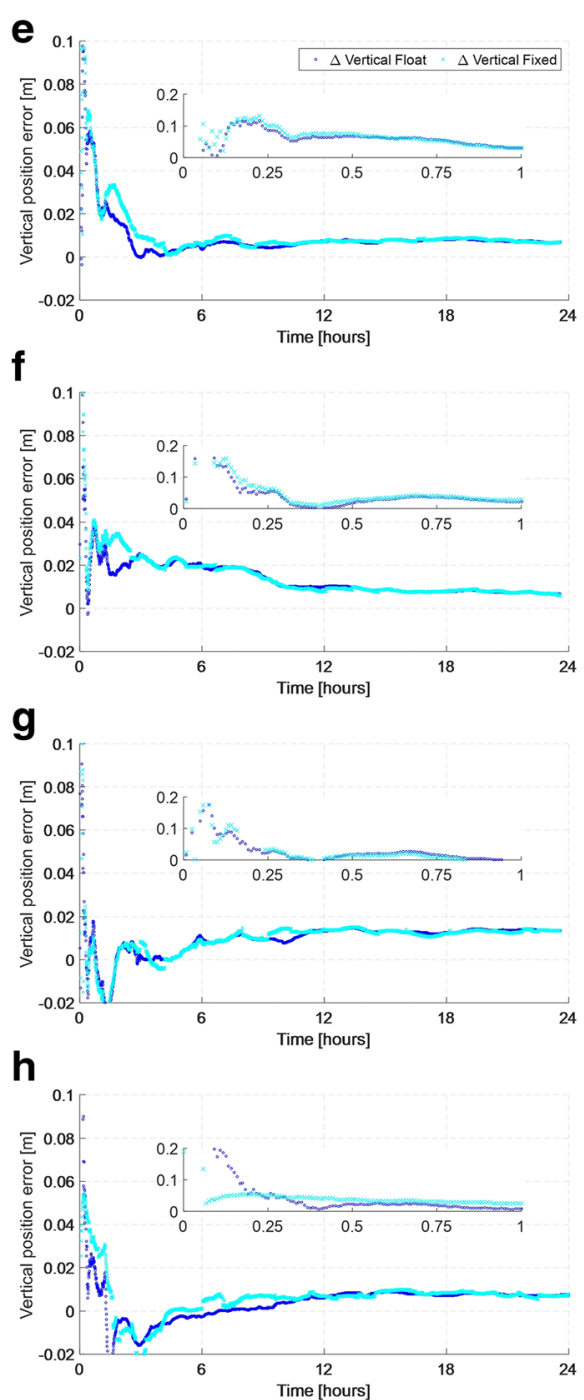

Fig. 11 Site ALGO DOY 28 of 2015 located in Algonquin, Canada, illustrating the differences between the "float" and "fixed" solution All units are in metres and different axis limits are utilized for horizontal and vertical subplots. a Horizontal component using the DC products. b Horizontal component using the transformed IRC (GRG) products. c Horizontal component using the transformed IRC (CNT) products. $\mathbf{d}$ Horizontal component using the transformed FCB products. e Vertical component using the DC products. $\mathbf{f}$ Vertical component using the transformed IRC (GRG) products. $\mathbf{g}$ Vertical component using the transformed IRC (CNT) products. $\mathbf{h}$ Vertical component using the transformed FCB products 
Presented in Table 1 was a summary of the different qualities of the products that were utilized within the study. IRC products were generated from a network of reference stations globally distributed, available in realtime and post-processed using final orbits and clocks. Similar to the IRC, the DC and FCB products were generated from a global network of solutions but postprocessed. Post-processed orbits and clocks have an accuracy of $\sim 2.5 \mathrm{~cm}$ and $\sim 75 \mathrm{ps}$, respectively, whereas the predicted half of ultra-rapid orbits and clocks have an accuracy of $\sim 5 \mathrm{~cm}$ and $\sim 3 \mathrm{~ns}$ respectively. While it is evident in existing literature PPP-AR is possible in realtime, the solution is more sensitive as the orbit and clocks have a lower accuracy and more susceptible to outages and network failures.

The general assumption when PPP products are estimated within the network, it is assumed that the PPP user would follow similar conventions when utilizing the products from the network. Consequences of different conventions adopted may result in incorrect ambiguities being resolved. For example, if inconsistent satellite antenna convention is adopted between the network and user, when phase wind-up corrections are applied, fractional cycles would be introduced. Presented in Fig. 12 is the orientation of the spacecraft body frame for GPS Block IIR/IIR-M satellites provided in the manufacturer specifications, subplot (a) and adopted within the IGS axis convention, subplot (b). The difference between the manufacturer specifications and IGS axis convention is the orientation of the X, Y - axis. For more details on the conventions for constellation-specific spacecraft body frames can be found in Montenbruck (2015).

Another critical component to be accounted for is the difference in the modelling of yaw maneuvers. Yaw maneuvers occur when the actual yaw angle differs from the nominal yaw angle. The nominal yaw angle is the orientation angle by which a satellite would maintain optimal solar visibility throughout its orbit, provided it could spin arbitrarily fast. The actual yaw angle is the orientation that the satellite can maintain due to its limited rate of yaw.

All satellites fail to maintain their nominal orientation when their orbits pass close to the Earth-Sun axis. These are the eclipsing orbits with turns at both orbit noon and orbit midnight. During a satellite eclipse, Block II GPS satellites behaved unpredictably because of hardware sensitivity, spinning beyond the nominal amount upon entering the sun's shadow. The Block IIR and Block IIF generations of satellites were designed to be able to maintain their nominal attitude even during orbit noon and orbit midnight (Bar-Sever 1996; Dilssner et al. 2011). For Block IIR, the yaw maneuver is constrained by a maximum yaw rate of 0.2 deg./s (Kouba 2009) and Block IIF is constrained by a maximum yaw rate of

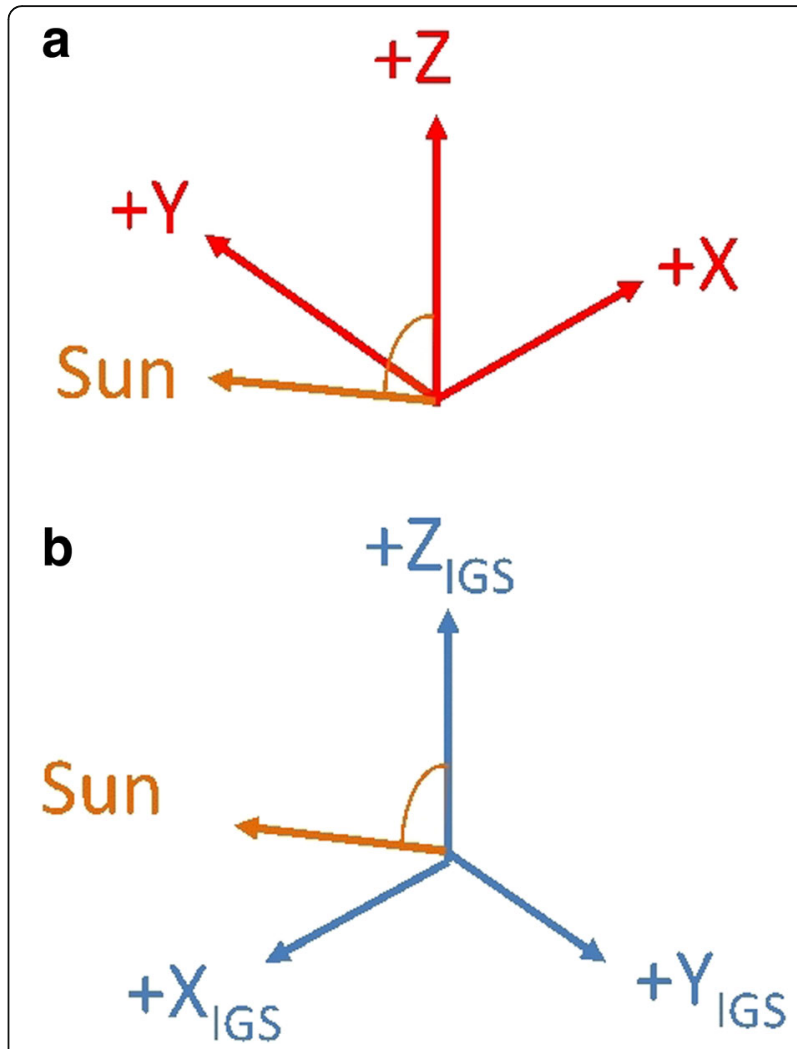

Fig. 12 Orientation of the spacecraft body frame for GPS Block IIR/IIRM satellites (Montenbruck et al. 2015). Sub-plot (a) refers to the manufacturer specification system while sub-plot (b) refers to the IGS axis conventions. a Manufacturer specifications. b IGS axis convention

0.11 deg./s (Dilssner 2010). The attitude model of the GPS satellites affects the computation of measurement geometry through variations of the transmitter phase center location and carrier-phase measurement wind-up. It also affects the modelling of the solar radiation pressure force acting on the GPS satellites due to the changes in illumination geometry (Kuang et al. 2016).

The expectation is that Block IIR and Block IIF satellites can maintain their nominal attitude even when orbiting through the penumbra and the umbra. As a result of the difficulties in determining the exact moment of exiting the umbra, modelling inconsistencies between ACs have been observed (Seepersad et al. 2016). In the DC format, for example, yaw angles and instances of orbit noon and midnight are provided. The expectation is all ACs would begin to provide this information to the PPP user to increase consistency between the network and user solution. If the networked defined periods of orbit noon, orbit midnight and yaw angles are not provided, it is recommended to the PPP user not to resolve ambiguities of satellites exiting the umbra if differences in yaw modelling exists between the network and user may exist. For more details on inconsistencies between ACs and combining PPP-AR products can be found in Seepersad et al. (2016). 


\section{Conclusions}

Interoperability of PPP-AR products is important, as it can increase the reliability of the user solution while offering similar performance, in regards to precision and accuracy. Interoperability of the products is possible for the PPP user, as the mathematical model, to enable an ambiguity resolved solution is similar. The different PPP-AR products contain the same information and would allow for a one-to-one transformation, allowing interoperability of the PPP-AR products. The PPP user will be able to transform independently generated PPP-AR products to seamlessly integrate within their PPP user solution. The seamless integration of the transformed products will allow the PPP user to have multiple solutions, which will increase the reliability of the solution, for e.g., real-time processing. During real-time PPP processing, if there was an outage in the generation of the PPP-AR products, the user can instantly switch streams to a different provider.

The three main public providers of products that enable PPP-AR were examined, which included School of Geodesy and Geomatics at Wuhan University (SGG-WHU), Natural Resources Canada (NRCan) and Centre National d'Etudes Spatiales (CNES). Equivalent performance was noted utilizing the different methods. Of the four solutions, FCB products had the highest accuracy. This is attributed to the products being generated using final IGS orbit and clock products. To confirm this, FCBs generated using GRG orbit and clock products were also examined and comparable performance was observed between the FCBs and IRC (GRG) products. The least accurate solution was obtained using the IRC (CNT) products, which was due to the products being archived real time products.

As the results indicated, interoperability of PPP-AR products is feasible. While feasible, there were challenges when processing the different PPP-AR products. These challenges were due to the same conventions not being followed between the network and user solution, for e.g. different satellite antenna convention. When different satellite antenna convention was used, fractional cycles was introduced when carrier-phase wind-up correction was applied. Another critical component to be accounted for is the difference in the modelling of yaw maneuvers. Difficulties in determining the exact moment when an eclipsing satellite exits the umbra, results in modelling inconsistencies between ACs. If network-defined periods of orbit noon, orbit midnight and yaw angles are not provided, it is recommended that the PPP user not attempt to resolve ambiguities of satellites exiting the umbra if differences in yaw modelling exists between the network and user.

\section{Future work}

Future work will consist of introducing a more advanced AR and validation technique; GPS L5; multi-constellation float and analysis of the post-fit residuals to examine the effects of mismodelling. The temporal and spatial behaviour of these estimated terms will be examined for the different products applied to understand the unmodelled effects that introduced incorrect solution fixes. The number of reference stations examined will also be increased to further test the reliability of the transformed products under varying user conditions.

\begin{abstract}
Authors informations
Garrett Seepersad is a Ph.D. candidate at York University, Toronto, Canada, in the Department of Earth and Space Science and Engineering. He has completed his B.Sc. in Geomatics at the University of West Indies and his M.Sc. in Geomatics Engineering at York University. His area of research currently focuses on the development and testing of PPP functional, stochastic and error mitigation models.

Dr. Sunil Bisnath is an Associate Professor in the Department of Earth and Space Science and Engineering at York University in Toronto, Canada. His research interests include GNSS processing algorithm development for positioning and navigation applications.
\end{abstract}

\section{Authors' contributions}

Both authors contributed equally in the writing and preparation of the manuscript. Both authors read and approved the final manuscript.

\section{Funding}

Funding was provided by Natural Sciences and Engineering Research Council of Canada (NSERC). NSERC aims to make Canada a country of discoverers and innovators for the benefit of all Canadians. The agency supports university students in their advanced studies, promotes and supports discovery research, and fosters innovation by encouraging Canadian companies to participate and invest in postsecondary research projects. NSERC researchers are on the vanguard of science, building on Canada's long tradition of scientific excellence.

\section{Competing interests}

The authors declare that they have no competing interests.

\section{Publisher's Note}

Springer Nature remains neutral with regard to jurisdictional claims in published maps and institutional affiliations.

Received: 9 April 2017 Accepted: 30 November 2017

Published online: 15 December 2017

\section{References}

Aggrey JE (2015) Multi-GNSS precise point positioning software architecture and analysis of GLONASS pseudorange biases. York University, Toronto

Bar-Sever YE (1996) A new model for GPS yaw attitude. J Geod 70:714-723

Bertiger W, Desai SD, Haines B et al (2010) Single receiver phase ambiguity resolution with GPS data. J Geod 84:327-337. doi: 10.1007/s00190-010-0371-9 CNES (2015) Le site du Centre national d'études spatiales. https://cnes.fr/

Collins P (2008) Isolating and estimating undifferenced GPS integer ambiguities. In: Proc. ION NTM. pp 720-732

Collins P, Bisnath S (2011) Issues in ambiguity resolution for precise point positioning. In: proceedings of the 24th international technical meeting of the satellite division of the Institute of Navigation (ION GNSS 2011). pp 679-687

Collins P, Bisnath S, Lahaye F, Héroux P (2010) Undifferenced GPS ambiguity resolution using the decoupled clock model and ambiguity datum fixing. Navigation 57:123-135

Collins P, Lahaye F, Heroux P, Bisnath S (2008) Precise point positioning with ambiguity resolution using the decoupled clock model. In: Proceedings of the 21st international technical meeting of the satellite division of the Institute of Navigation (ION GNSS 2008). pp 1315-1322.

Dilssner F (2010) GPS IIF-1 satellite antenna phase center and attitude modeling. GNSS 5:59-64

Dilssner F, Springer T, Gienger G, Dow J (2011) The GLONASS-M satellite yaw-attitude model. Adv Space Res 47:160-171. doi: 10.1016/j.asr.2010.09.007 
Ge M, Gendt G, Rothacher M et al (2008) Resolution of GPS carrier-phase ambiguities in precise point positioning (PPP) with daily observations. J Geod 82:389-399. doi: 10.1007/s00190-007-0187-4

Geng J (2010) Rapid integer ambiguity resolution in GPS precise point positioning. University of Nottingham

Geng J, Meng X, Dodson AH, Teferle FN (2010) Integer ambiguity resolution in precise point positioning: method comparison. J Geod 84:569-581. doi: 10. 1007/s00190-010-0399-x

Geng J, Shi C, Ge M et al (2012) Improving the estimation of fractional-cycle biases for ambiguity resolution in precise point positioning. J Geod 86:579-589

Geng J, Teferle FN, Shi C et al (2009) Ambiguity resolution in precise point positioning with hourly data. GPS Solut 13:263-270. doi: 10.1007/s10291-009-0119-2

Kouba J (2009) A simplified yaw-attitude model for eclipsing GPS satellites. GPS Solut 13:1-12. doi: 10.1007/s10291-008-0092-1

Kuang D, Desai S, Sibois A (2016) Observed features of GPS block IIF satellite yaw maneuvers and corresponding modeling. GPS Solut. doi: 10.1007/s10291016-0562-9

Lannes A, Prieur J-L (2013) Calibration of the clock-phase biases of GNSS networks: the closure-ambiguity approach. J Geod 87:709-731. doi: 10.1007/ s00190-013-0641-4

Laurichesse D (2014) Phase biases for ambiguity resolution: from an undifferenced to an uncombined formulation, http://ppp-wizard.net/Articles/ WhitePaperL5.pdf, Accessed 2 Jun 2015

Laurichesse D, Mercier F (2007) Integer ambiguity resolution on undifferenced GPS phase measurements and its application to PPP. pp 839-848

Laurichesse D, Mercier F, Berthias J-P et al (2009) Integer ambiguity resolution on undifferenced GPS phase measurements and its application to PPP and satellite precise orbit determination. Navigation 56:135-149

Li P, Zhang X, Ren X, et al (2015) Generating GPS satellite fractional cycle bias for ambiguity-fixed precise point positioning. GPS Solut doi: 10.1007/s10291-015-0483-z

Mercier F, Laurichesse D (2007) receiver/payload hardware biases stability requirements for undifferenced Widelane ambiguity blocking. In: Scientifics and fundamental aspects of the Galileo program Colloquium, Fall 2007

Mervart L, Lukes Z, Rocken C, Iwabuchi T (2008) Precise point positioning with ambiguity resolution in real-time. In: Proceedings of ION GNSS. pp 397-405

Montenbruck O, Schmid R, Mercier F et al (2015) GNSS satellite geometry and attitude models. Adv Space Res 56:1015-1029. doi: 10.1016/j.asr.2015.06.019

NRCan (2015) Natural Resources Canada. https://uww.nrcan.gc.ca. Accessed 19 Jun 2015

Schaer S (2016) SINEX BIAS - solution (software/technique) independent exchange format for GNSS BIASes

Scherneck H (2013) Ocean Tide Loading Provider. http://holt.oso.chalmers.se/ loading/. Accessed 2 Jan 2013

Seepersad G (2012) Reduction of initial convergence period in GPS PPP data processing. York University, Toronto

Seepersad G, Banville S, Collins P, et al (2016) Integer satellite clock combination for Precise Point Positioning with ambiguity resolution. In: Proceedings of the 29th International Technical Meeting of The Satellite Division of the Institute of Navigation (ION GNSS+ 2016), Portland, OR

Shi J, Gao Y (2013) A comparison of three PPP integer ambiguity resolution methods. GPS Solut 18:519-528. doi: 10.1007/s10291-013-0348-2

Shi J, Gao Y (2014) A troposphere constraint method to improve PPP ambiguityresolved height solution. J Navig 67:249-262. doi: 10.1017/S0373463313000647

Teunissen PJ, Odijk D, Zhang B (2010) PPP-RTK: results of CORS network-based PPP with integer ambiguity resolution. J Aeronaut Astronaut Aviat Ser A 42:223-230

Teunissen PJG, Khodabandeh A (2015) Review and principles of PPP-RTK methods. J Geod 89:217-240. doi: 10.1007/s00190-014-0771-3

Wuhan University (2017) School Of Geodesy and Geomatics,Wuhan University. In: Wuhan Univ. http://en.sgg.whu.edu.cn/. Accessed 19 Aug 2017

Zhang B, Teunissen PJG, Odijk D (2011) A novel un-differenced PPP-RTK concept. J Navig 64:S180-S191. doi: 10.1017/S0373463311000361

\section{Submit your manuscript to a SpringerOpen ${ }^{\circ}$ journal and benefit from:}

- Convenient online submission

- Rigorous peer review

- Open access: articles freely available online

- High visibility within the field

Retaining the copyright to your article

Submit your next manuscript at springeropen.com 